\title{
Evaluation of Robustness, Accuracy and Speed of SPIRAL for Fast Fingerprint Biometric System
}

\author{
Rahul Sharma \\ CSE Department \\ Krishna Institute of Engineering \\ \& Technology, Ghaziabad \\ UP, India
}

\author{
Nidhi Mishra \\ CSE Department \\ Shri Krishna Yogi Raj \\ Technical Institute, Hathras \\ UP, India
}

\author{
Sanjeev Kumar \\ CSE Department \\ Krishna Institute of Engineering \\ \& Technology, Ghaziabad \\ UP, India
}

\begin{abstract}
Fingerprint is most popular and common biometric trait used for authentication. The fingerprint is a physiological biometric characteristic to identify person. Fingerprint recognition is the problem of searching a fingerprint in the reference database to find a fingerprint that matches a given fingerprint. For enhancing the performance and accuracy of biometric fingerprint recognition system one of the faster technique SPIRAL proposed by Woon Ho Jung is evaluated for robustness, accuracy \& speed with varying constraints. To test robustness of authentication mechanism, different noises are added to original fingerprint images and found that speed \& accuracy of SPIRAL is remarkable but not robustness.
\end{abstract}

\section{Keywords}

Biometric, Fingerprint Identification, Noise, SPIRAL, Wavelet Decomposition, Biometric System.

\section{INTRODUCTION}

Fingerprints are raised ridges of skin on the hairless surfaces of hands and feet (Dermal Ridges).Primates and other animals have fingerprints. They provide traction and every ridge contains glands [1]. Fingerprint Identification is the method of identification using the impressions made by the minute ridge formations or patterns found on the fingertips. There are few desirable properties for chosen fingerprint biometric characteristic [10]. These include universality, uniqueness, collectability and acceptability: The user population and the public in general do not have objections to the measuring/collection of the biometric trait.

A biometric system defined as pattern matching system where we acquire and store data of individuals in database and compare expected features against the stored template authenticate. [11]

Biometrics is a science which is used to of verifying the identity often individual through physiological measurements or behavioural traits. Fingerprint recognition is a famous biometric system mainly because of simplicity in acquisition. In general every person has ten fingers so availability of biometric trait becomes easier. At present time the automatic fingerprint recognition systems has drawn special attention due to its performance over other biometric systems. We have challenges to make faster with accuracy for fingerprint authentication [8].

The main aim of this paper is develop a Fingerprint Recognition System that returns relevant results to a query fingerprint image in a relevant time and evaluate SPIRAL technique under different constraints like different noises. All implementation work is done in MATLAB.

\section{LITERATURE}

In twentieth Century fingerprints are used for criminal identification. These fingerprints also used for prisoners identification in New York at that time.

In 14th century, In China the first known example of biometrics was a form of fingerprinting being used as reported by explorer Joao de Barros [1] in 1880.Various researchers shown interest in the field of fingerprint recognition system.

Authors in [7] proposed a non-minutiae algorithm for the fingerprint authentication. This algorithm is based on discrete wavelet transformation (DWT) which decomposes an input fingerprint into sub bands of LL, LH, $\mathrm{HL}$ and HH.An angle and Coherence are calculated on LL band only.

Authors in [5] use the fingerprint image-intensity domain. A correlation filter is applied on this domain and finds the wavelet spaces for a fingerprint class that are most conducive to correlation filter recognition.

Authors in [9] propose algorithm consists of 2 stages, The training stage is performed off-line thus it is not very sensitive in terms of runtime. The verification stage is performed online therefore it needs to be as fast as possible.

Authors in [3] proposed SPIRAL technique for making recognition system fast. SPIRAL generates software (SW) implementations for linear digital signal processing (DSP) transforms that are tuned to target hardware (HW) platform. Author addressed a high level SPIRAL's approach of finding fast code among the possible alternatives.

\section{SPIRAL TECHNIQUE}

SPIRAL: SPIRAL is stand for signal processing implementation research for adaptive libraries. It generates fast implementation for linear DSP transform. The SPIRAL leads to "fast" algorithm. The term 'fast' refers to the operations count. It is number of operations required to compute the transform.

SPIRAL provides some unique properties:-

i. SPIRAL is applicable to the entire domain of linear digital signal processing algorithms, and this domain encompasses a large class of mathematically complex algorithms.

ii. SPIRAL encapsulates the mathematical algorithmic in a concise declarative framework suitable for computer representation, exploration, and optimization.

iii. SPIRAL can be expanded in several directions to include new transforms, new optimization techniques, different target performance metrics, and a wide variety of implementation platforms. 


\subsection{The Architecture of SPIRAL}

SPIRAL's design is based on the following assumption [4]:

i. Many possible fast algorithms can be represented as formulas in a concise mathematical notation using a small number of mathematical constructs and primitives.

ii. The automatically generated algorithms can be automatically translated into a high-level language (such as $\mathrm{C}$ etc) program.

Based on these facts, SPIRAL translates the task of finding hardware adapted implementations into an intelligent search in the space of possible fast algorithms and their implementations.

The two basic steps that SPIRAL takes to go from the highlevel specification of the transform $\mathrm{Tn}$ to an actual implementation I $€$ I of Tn. The two steps correspond:

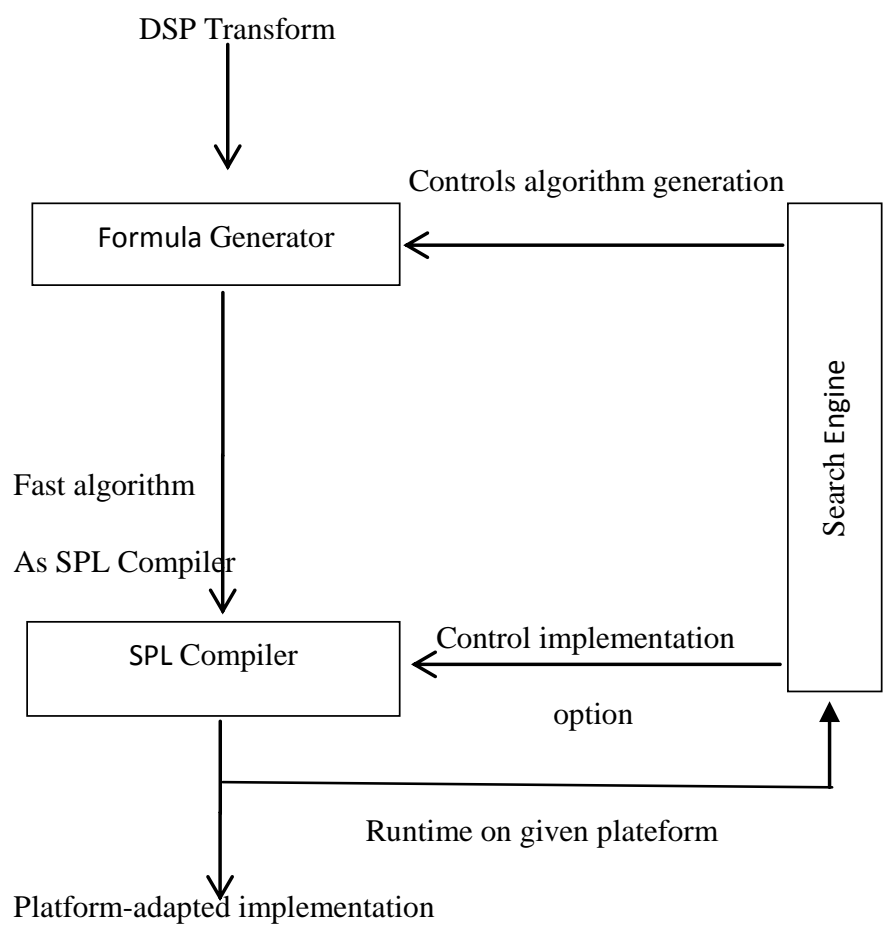

Fig.1: The Architecture of SPIRAL [4]

\section{SYSTEM IMPLEMENTATION}

This work, Evaluation of SPIRAL for Fast Fingerprint Recognition System uses the three main modules.

\subsection{Training stage}

In this step trained the input images of fingerprints of particular size $(128 * 128)$ and format (.bmp) are stored in database. The training stage is performed off-line thus it is not very sensitive in terms of runtime because the end users will not suffer from it[19]. In this stage we train the input image. Steps are following-

Step 1 - Full decomposition of input image- Decomposition of an image is done by wavelet packet decomposition [6]. By applying the wavelet packet decomposition, four subspaces of the original image is obtained. Applying recursively the same routine to the child nodes until a full tree with height equal to 4 is constructed.
Step 2 - Generate score tree- In this step we generate the score tree by combining the data stored in the nodes of tree. The root of all the trees in the tree list generated in the full decomposition step is needed to generate the root of the score tree. The score tree contains the Fitness score of each subspace in the nodes of the tree. The fitness score is computed by the following formula:

$$
\begin{aligned}
& \text { Fitness Formula }=\frac{1}{\left.\mathrm{U}^{\mathrm{T}}\left(\mathrm{X}^{+} \mathrm{D}^{-1} \mathrm{X}\right)^{-1} \mathrm{U}\right)}
\end{aligned}
$$

Where

$\mathrm{X}=\quad$ [DWT(img1),DWT(img2),.........DWT(imgn) $]$

Each column of matrix $\mathrm{X}$ holds the spectrum of one of the training images after projecting it onto the wavelet subspace. $\mathrm{X}^{+}=$complex conjugate of $\mathrm{X}$.

$\mathrm{D}=$ diagonal matrix with the average power spectrum of the training images along its diagonal.

$\mathrm{U}=$ column matrix with all ones.

Step 3 - Pruning of score tree- Pruning is used to prune the score tree based on the fitness score stored in each node of the score tree which is generated in previous step. In pruning step, we have remained with only nodes or subspaces which having the better score than the parent node.

Step 4 - Compute the correlation filter- In this step compute the correlation filter for the nodes or subspaces that are located at the leaves of the score tree. The correlation filter is computed by the following formula:--

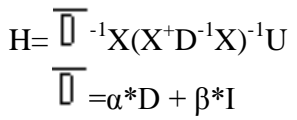

$\alpha$ and $\beta$ are constants parameters between 1 and 0 .

Both the correlation filter and the score tree in each node of the pruned score tree is saved.

\subsection{Verification Stage}

The verification stage is performed on-line therefore it needs to be as fast as possible. [9] The quality of a fingerprint identification system not only depends on the time that it takes to compute the answer. Steps involved in verification stage are as follows:

Step1-Decompose input fingerprint Image- The decomposition of the input image in the verification is similar to the one in the training stage. The fingerprint image is decomposed into the subspace that is matched with the pruned tree of the training stage.

Step 2- Applying wavelet transform- In this step, apply DWT (Discrete Wavelet Transform) to each of the subspaces or node obtained in the decompose input fingerprint image step.

Step 3 - Point wise multiplication- As we obtained correlation filter in the training stage, we apply point wise multiplication with the matrix produced in the previous step.

Step 4 - Apply Inverse Wavelet transform- after obtaining the new pointwise multiplication metrics, we apply inverse discrete wavelet transformation (IDWT) to each of the subspaces obtained to the new matrix produced in the Point wise Multiplication step. 
Step 5 - Peaks Verification- This step shows the fingerprint authentication. If the input fingerprint is authenticated then high peak should be present in the matrix.

\subsection{SPIRAL Function}

The verification stage is performed on-line therefore it needs to be as fast as possible. So, the runtime of the verification stage is very important. Therefore, SPIRAL technique is used to automatically generate and optimize code for each distinct fingerprint image. SPIRAL takes an mathematical expression and automatically generate code that implements the given expression. In the training routine, a mathematical expression that describe the user score tree and call SPIRAL with the derived expression in order to generate the code for the verification stage .SPIRAL has ability to automatically generate a verification routine that is specifically implemented and optimized for each user and the platform that the system is running on[9].

The verification algorithm having two building blocks:-

- $\operatorname{MDH}(\mathrm{n})$

- $\operatorname{MDDWT}(\mathrm{n})$

MDDWT(n) represent the leave nodes of the score tree.

MDH(n) represent the other intermediate nodes of the score tree.

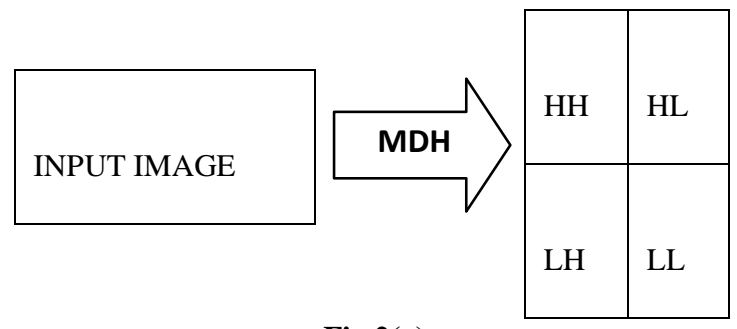

Fig.2(a)

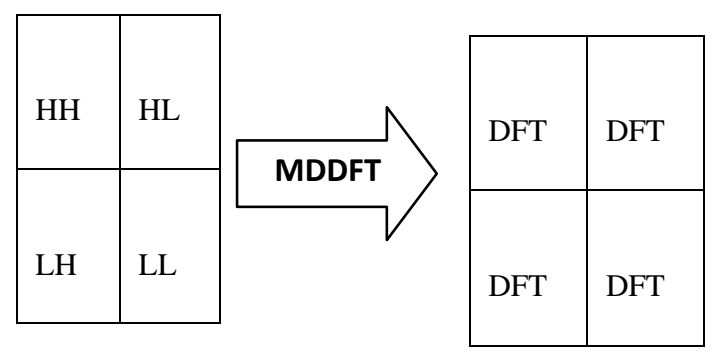

Fig.2 (b)

Fig.2 (a). Image splits into subspaces and (b) MDDFT(n)applies multidimensional DFT to each subspace.

The simple rule of the SPIRAL is applied recursively to generate the full expression:-.

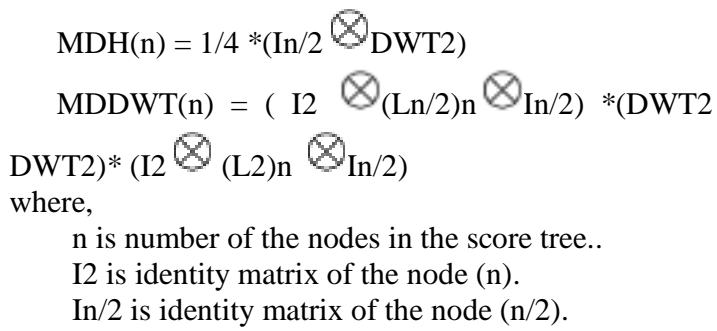

DWT2 is Discrete Wavelet Transform in MATLAB code.

$(\mathrm{Ln} / 2) \mathrm{n}$ is Stride Permutation matrix.

Stride Permutation matrix (Lk)n is a which read the input at the stride $\mathrm{k}$ and storeit at stride 1,defined by its corresponding permutation:-

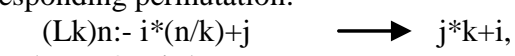

where, $0<=\mathrm{i}<\mathrm{k}$;

$0<=\mathrm{j}<\mathrm{n} / \mathrm{k}$;

In the above equation, $\mathrm{k}$ is equal to 2 .

is Kronecker Tensor Product

Syntax:-K = kron $(\mathrm{X}, \mathrm{Y})$

Description: $-\mathrm{K}=\operatorname{kron}(\mathrm{X}, \mathrm{Y})$ returns the Kronecker tensor product of $\mathrm{X}$ and $\mathrm{Y}$. The result is a large array formed by taking all possible products between the elements of $X$ and those of $\mathrm{Y}$. If $\mathrm{X}$ is $\mathrm{m}-\mathrm{by}-\mathrm{n}$ and $\mathrm{Y}$ is $\mathrm{p}-\mathrm{by}-\mathrm{q}$, then $\operatorname{kron}(\mathrm{X}, \mathrm{Y})$ is m*p-by-n*q.[10].

\section{RESULTS AND DISCUSSION}

First we take a fingerprint as input for the training stage. This image is of $128 * 128$ in dimension and $17.0 \mathrm{~KB}$ in size and having .bmp format.

Table 1.Database of Four Fingerprints

\begin{tabular}{|c|c|c|}
\hline S.No & $\begin{array}{l}\text { Image } \\
\text { Name }\end{array}$ & Images \\
\hline 1. & I1.bmp & \\
\hline 2. & I2.bmp & \\
\hline 3. & I3.bmp & \\
\hline 4. & I4.bmp & \\
\hline & & \\
\hline
\end{tabular}

In training Stage, following figure is obtained. In this figure first four columns shows the decomposition of the four fingerprints. Each image start decomposed from 128*128 dimensions to $16^{*} 16$ dimensions. Fifth column contains the fitness score value.Sixth column represents the value 0 and 1.The node which having the maximum score is indicated as 1 and if its value is less than it is 0 .Seventh column represent the correlation filter value where one is shown in fifth column. 


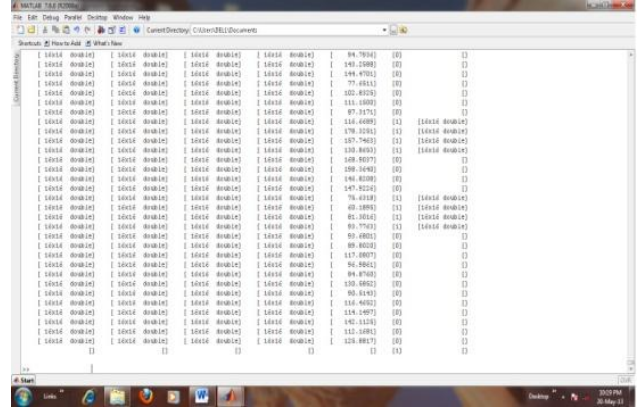

Fig.3. Training Stage of Four Fingerprint Images

The graph is plotted using meshgrid and the graph which shows the single peak are the images that are in the database. So, there graphs having the single peak, hence these images are in database. Here we consider first two fingerprint images for authentication and we get following graph.

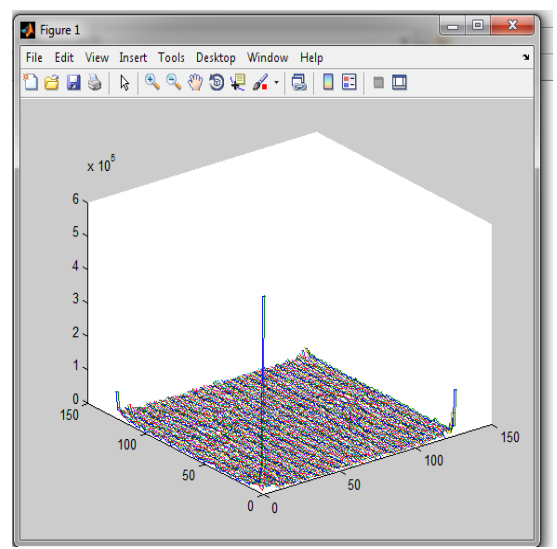

Fig.4. Peaks for I1.bmP

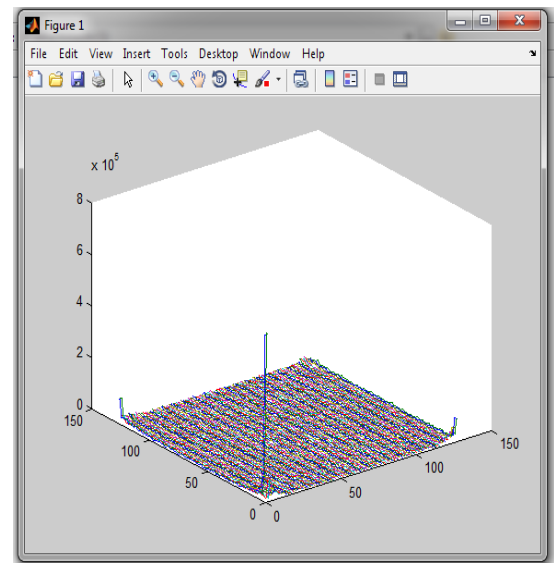

Fig.5. Peaks for I2.bmp

\subsection{Result by Using Spiral Technique}

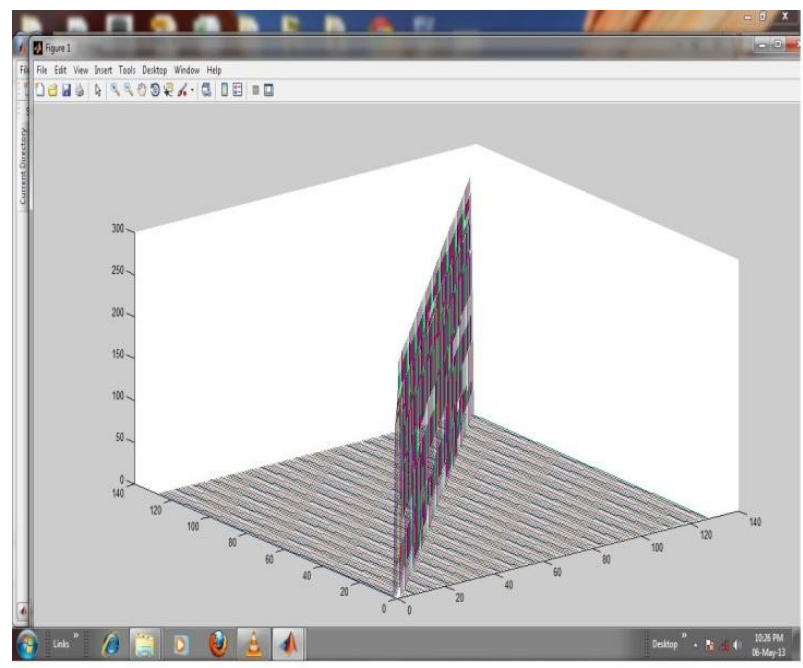

Fig.6. Graph Showing the SPIRAL Function Used in the Verification Stage.

\subsection{Graphs for Unauthenticated Images}

If the graph does not contain the single peak it shows that the image is not in the database .Since, these graphs have low peaks; hence these images are not authenticated. We consider a fingerprint which is not in database like I5.bmp.then following graph is obtained.

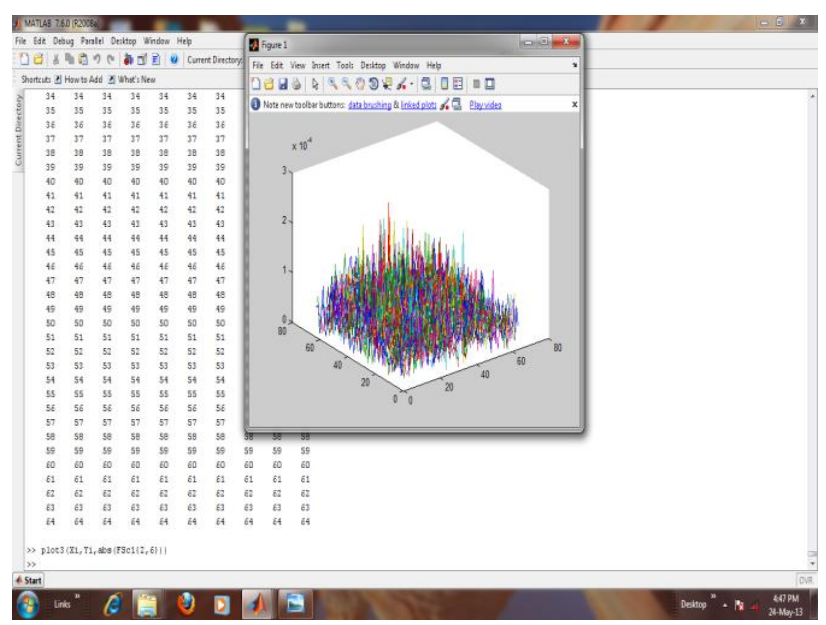

Fig.7. Peaks for Image that are not in Databae

\subsection{Add Noise in Fingerprints}

If we add noise such as Gaussian, Poisson, Speckle Noise in the fingerprint then graph does not contain single peak. We insert the Gaussian noise in I1.bmp then we get the following graph. 


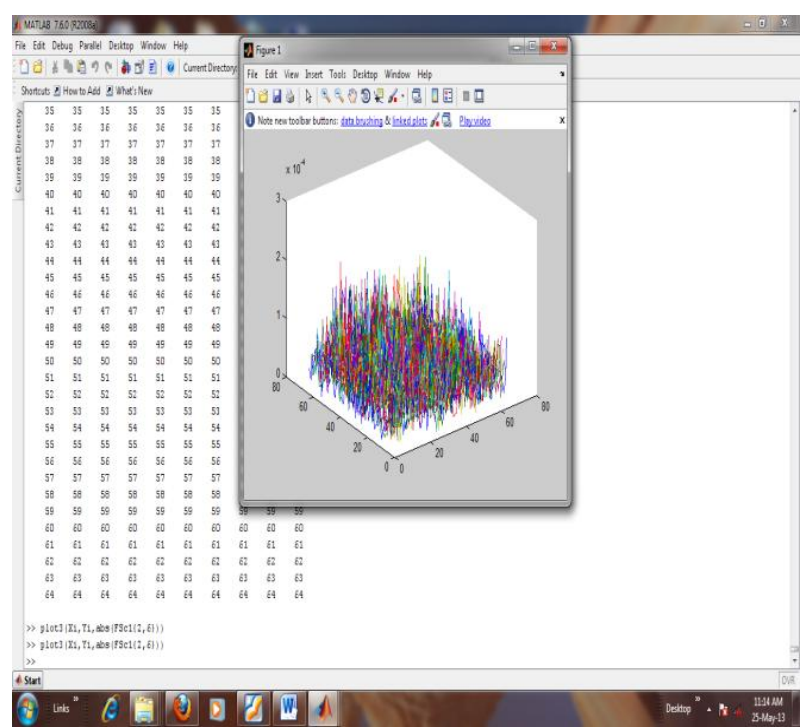

Fig.7.Graph for I1.bmp fingerprint with gaussian Noise

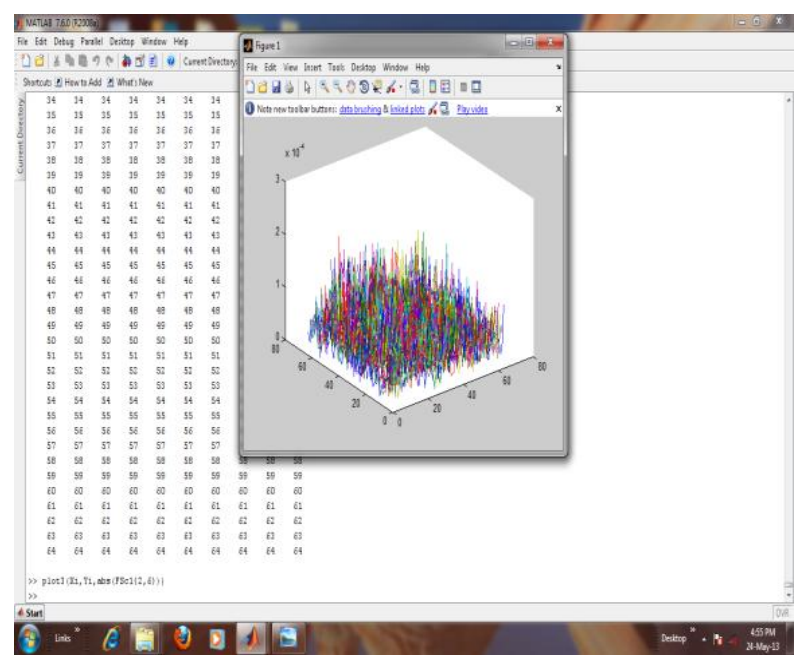

Fig.8.Graph for I1.bmp fingerprint with Poisson Noise

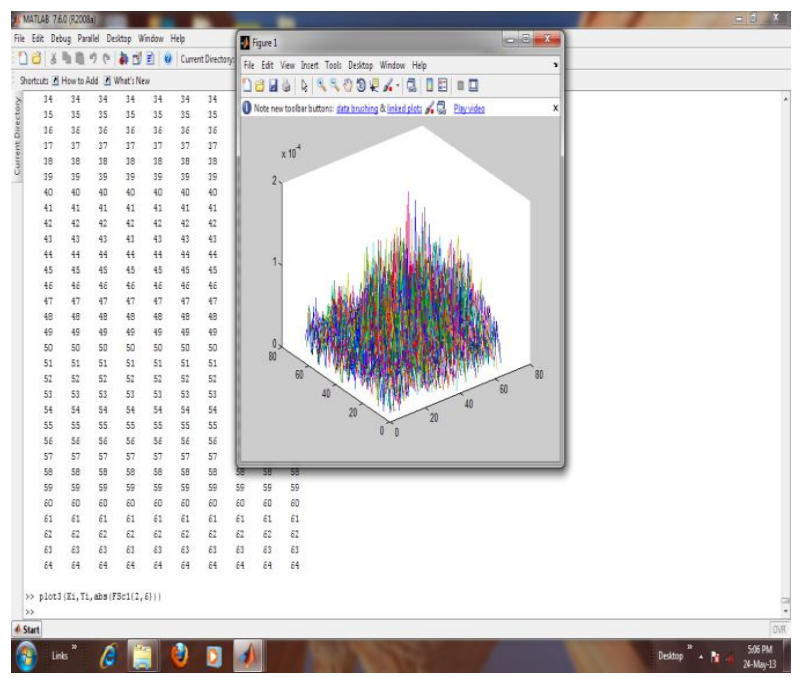

Fig.9.Graph for I3.bmp fingerprint with Speckle Noise

\subsection{Time Analysis}

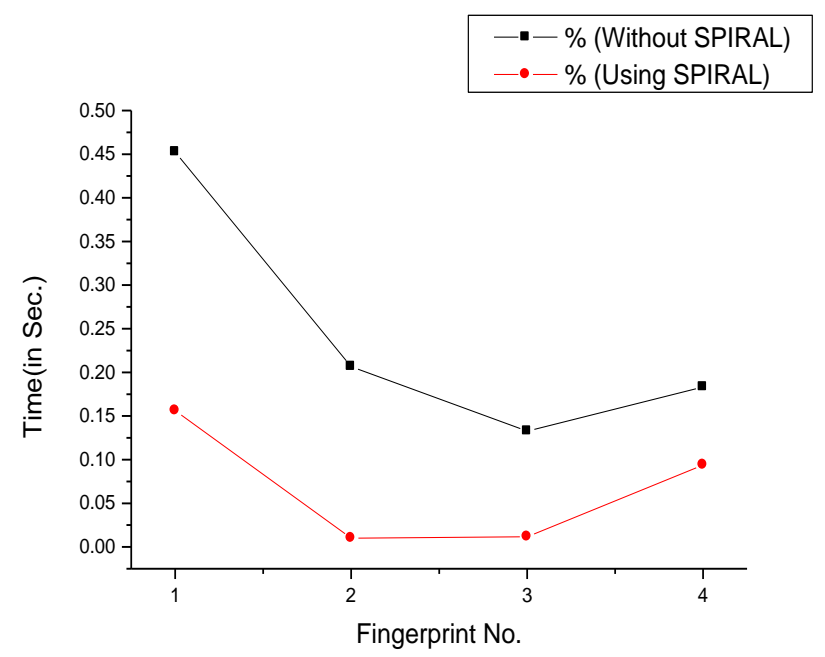

Fig.10. Graph showing the time analysis for the original fingerprints with SPIRAL and without SPIRAL

\section{CONCLUSION AND FUTURE SCOPE}

In this work, we implemented SPIRAL technique to authenticate fingerprint. Authentication is done in two stages:Training Stage and Verification Stage. SPIRAL is known for fast recognition of fingerprint images and found to be satisfactory in performance. Second objective of this work was evaluating robustness of SPIRAL when gaussion, poisson and speckle noises were introduced in input images but SPIRAL is not found to authenticate given images so SPIRAL is not robust while in all stages it was faster.

As per results achieved during testing robustness of SPIRAL time is one possibility of future work is the enhance SPIRAL so it can work efficiently with noisy images noise too. SPIRAL is outstanding in speed and accuracy but improving robustness would make it a remarkable technique for fingerprint biometric system.

\section{REFERENCES}

[1] António Farelo,2005, A History of Fingerprints, INTERPOL.

[2] Markus P"uschel, Adam C. Zelinski, and James C. Hoe,2004,Custom-Optimized Multiplier less Implementations of DSP Algorithms,IEEE.

[3] Markus Puschel, Jose M. F. Moura, Jeremy Johnson, David Padua, Manuela Veloso, Bryan W. Singer, JianxinXiong, Franz Franchetti, AcaGaci'c, YevgenVoronenko, Kang Chen, Robert W. Johnson, Nicholas Rizzolo,2005, SPIRAL: Code Generation for DSP Transforms,Proceedings of the IEEE, special issue on "Program Generation, Optimization And Adaptation", Vol. 93, pp. 232- 275.

[4] Markus Puschel, Jose M. F. Moura, Jeremy Johnson, David Padua, Manuela Veloso,RobertW.Jhonson et al,2004,SPIRAL:a Generator for Plateform-Adapted Libraries of the Signal Processing Algorithms.,IJHPCA,Vol.18,No.1,pp 21-45. 
[5] Pablo Hennings, Jason Thornton, JelenaKovacevic, and B. V. K. Vijaya Kumar, Feb. 2005 ,Wavelet packet correlation methods in biometrics, Applied Optics, Volume.44, Issues 5, , pp. 637-646.

[6] Paul Schrater, 2009, Recognizing Characters in Natural Scene: A Feature Study, CSci 5521 Pattern Recognition.

[7] Shashi Kumar D R, K B Raja, R K Chhotaray, Sabyasachi Pattanaik,2011,DWT Based Fingerprint Recognition using Non Minutiae Features,IJCSI International Journal of Computer Science Issues, Vol. 8, Issue 2 .

[8] Shila Samantaray,2011,ADoG based approach for fingerprint Image Enhancemment, Thesis submitted at NIT,Rourkela.
[9] Woon Ho Jung,2005, Fast Fingerprint Recognition Using Spiral, Department of Electrical and Computer Engineering, Carnegie Mellon University.

[10] Wrzeczycki8. Franz Franchetti ,2006 ,Stride Permutations and Tensor Products, Volume 4395 of LNCS., Springer,pp 363-377.

[11] X.D. Jiang, W.Y. Yau, 2000, Fingerprint Minutiae Matching Based on the Local and Global Structures, in Proc. 15th ICPR. Barcelona, Spain, pp. 1038-104.

[12] Peters, R.A ,1995,A New Algorithm for Image Noise Reduction using Mathematical morphology,IEEE Transaction on Communication V.4,No. 5,554-568.

[13] Rafael C. Gonzalez, 2008, Digital Image Processing, Edition-3,Pearson Education India. 